\title{
Neuronal vacuolation and spinocerebellar degeneration associated with altered neurotransmission
}

\author{
Aggeliki Giannakopoulou \\ Laboratory of Anatomy, Histology and Embryology, School of Veterinary Medicine, Faculty of Health Sciences, Aristotle University \\ of Thessaloniki, Thessaloniki, Greece and Department of Hematology, G. Papanikolaou General Hospital, Thessaloniki, Greece
}

\begin{abstract}
Inherited neurodegenerative disorders are debilitating diseases that occur across different species, such as the domestic dog (Canis lupus familiaris), and many are caused by mutations in the same genes as corresponding human conditions. In the present study, we report an inherited neurodegenerative condition, termed 'neuronal vacuolation and spinocerebellar degeneration' (NVSD) which affects neonatal or young dogs, mainly Rottweilers, which recently has been linked with the homozygosity for the RAB3GAP1:c.743delC allele. Mutations in human RAB3GAP1 cause Warburg micro syndrome (WARBM), a severe developmental disorder characterized predominantly by abnormalities of the nervous system including axonal peripheral neuropathy. RAB3GAP1 encodes the catalytic subunit of a GTPase activator protein and guanine exchange factor for Rab3 and Rab18 proteins, respectively. Rab proteins are involved in membrane trafficking in the endoplasmic reticulum, autophagy, axonal transport and synaptic transmission.

The present study attempts to carry out a detailed histopathological examination of NVSD disease, extending from peripheral nerves to lower brain structures focusing on the neurotransmitter alterations noted in the cerebellum, the major structure affected. NVSD dogs presented with progressive cerebellar ataxia and some clinical manifestations that recapitulate the WARBM phenotype. Neuropathological examination revealed dystrophic axons, neurodegeneration and intracellular vacuolization in specific nuclei. In the cerebellum, severe vacuolation of cerebellar nuclei neurons, atrophy of Purkinje cells, and diminishing of GABAergic and glutamatergic fibres constitute the most striking lesions. The balance of evidence suggests that the neuropathological lesions are a reaction to the altered neurotransmission. The canine phenotype could serve as a model to delineate the disease-causing pathological mechanisms in RAB3GAP1 mutation.
\end{abstract}

Key words: spinocerebellar ataxia, Purkinje cells atrophy, neurotransmitters, neurodegeneration.

\section{Introduction}

Several inherited disorders of men are characterized by progressive cerebellar degeneration, but attempts to correlate the observed clinical signs with the neuropathological lesions are complicated by problems associated with the analysis of human post-mortem material [49]. Moreover, in most disorders, the relationship between the appearance of a lesion and the underlying disease process is unclear [5]. Recently, a well-documented inherited disease of Rottweiler dogs named neuronal vacuolation spi- 
nocerebellar degeneration (NVSD) has been linked with the homozygosity for the RAB3GAP1:c.743delC allele [32]. Mutations in human RAB3GAP1 cause Warburg micro syndrome (WARBM), a severe developmental disorder characterized predominantly by abnormalities of the nervous system including axonal peripheral neuropathy. In WARBM and NVSD, the effect of gene mutation encoding RAB3GAP1 on the degeneration of specific anatomical pathways, has not yet been clarified.

WARBM is a rare autosomal recessive genetic disorder, caused by changes in one of at least four different genes, RAB3GAP1, RAB3GAP2, RAB18 and $T B C 1 D 20$. In relation to $R A B 3 G A P 1$, which is also mutated in NVSD, is a $130-\mathrm{kDa}$ protein that forms, together with the $150 \mathrm{kDa}$ RAB3GAP2, the heterodimeric RAB3GAP complex. This complex regulates the activity of members of the RAB3 family that are master regulators of vesicle trafficking and exocytosis of hormones and neurotransmitters [20,39]. RAB family members cycle between a GDP-bound inactive and a GTP-bound active form. The GTP-bound active form of RAB3 family members is inactivated by GTP hydrolysis before, during, and after the fusion of the vesicle by the stimulation of RAB3GAP [14]. Rab3GAP not only functions as a GAP for the Rab3s but also functions as a guanine exchange factor (GEF) for Rab18. Specifically, Rab3GAP localizes to the endoplasmatic reticulum (ER) and some point mutations in RAB3GAP1 that cause WARBM in humans affect Rab18 GEF activity [16]. Supporting the view that Rab18 activity is important for the ER structure, direct loss of Rab18 function or loss of Rab18 activation at the ER by the absence of Rab3GAP activity can cause WARBM [19].

WARBM and NVSD share considerable clinical similarities, although some differences do exist. NVSD affects puppies younger than 6 months, often as young as 6 to 8 weeks and sometimes littermates. It has been recognised mainly in Rottweiler dogs $[13,27,41,43,52]$ and their cross [12]. Typical signs of the disease include progressive cerebellar ataxia, spastic tetraparesis, dysmetria, ocular motor disorders such as episodic nystagmus, microphthalmia and congenital cataracts, axonal peripheral neuropathy with laryngeal paralysis and rarely behavioural changes $[27,30]$. Children with WARBM have severe developmental delays, ocular abnormalities including congenital cataracts and microphthalmia, and a predominantly axonal peripheral neuropathy and never develop the ability to walk $[18,20,38]$. Histopathological hallmarks in NVSD, which have been described so far, are the bilateral and symmetrical neuronal vacuolation involving brainstem and deep cerebellar nuclei (DCN), the Purkinje cell (PC) atrophy, the degeneration of the dorsolateral fasciculi in the spinal cord (SC) and the axonal neuropathy in peripheral nerves with spheroid formation and loss of thick myelinated fibres. There are no reports describing histopathology in WARBM, but MRIs of affected children have shown predominantly cerebellar atrophy $[20,33]$.

The present study investigates the four key features mentioned below, proposed by Armstrong [4] as the 'primary determinants' of a neurodegenerative disease, to provide a descriptive framework of NVSD. These features are the anatomical pathways affected by the disease, the target cell populations, the molecular pathology and the morphological degeneration, which are used to reveal the similarities and differences between NVSD and WARBM. The main focus of this study was the identification of neurotransmitter disturbances noted in the cerebellum of NVSDaffected animals, to clarify the underlying mechanisms involved. Herein we provide a comprehensive histological picture of NVSD and we suggest that the specific neuropathology is a reaction to degenerative processes due to altered neurotransmission caused by the RAB3GAP1 mutation.

\section{Material and methods Phenotypes}

Animal experimentation received the approval of the Veterinary Directorate of Thessaloniki and was conducted under compliance with the National Institutes of Health guidelines, Greek Government guidelines and the local ethics committee. The first dog was a 3-month-old female Rottweiler, which was initially admitted with acute inspiratory dyspnoea and stridor, both of which resolved after treating the animal in the intensive care unit. Neurological examination revealed ataxia, spastic tetraparesis, hypermetria and proprioceptive deficits, hyperreflexia and hypertonicity that were more pronounced in the hind limbs.

One month later, its littermate (second dog), a fourmonth-old male was also admitted with ataxia and weakness as the main complaints, as well as abnormal swallowing and failure to bark. Mild inspiratory stridor, ataxia, spastic tetraparesis, hypermetria, positional strabismus and nystagmus were detected in neuro- 
logical examination. A unilateral laryngeal paresis was diagnosed with laryngoscopy. Motor signs worsened progressively over the next two months in both puppies, finally leading to severe tetraparesis. Due to the severely incapacitating signs and poor prognosis, the puppies were eventually euthanized.

The third dog was a 6-month-old male from the same progenitors, which was presented with the wide base posture, hind limb weakness progressing to paraparesis and difficulty in swallowing and breathing associated to megaesophagus. This led to the compression of the trachea and difficulty in breathing. Motor signs worsened progressively over the next month leading to ataxia, spastic tetraparesis (more severe in hind limbs) with slowed proprioceptive placing reactions in all neurologically examined limbs. The breeder declined further examinations and requested euthanasia because of poor prognosis.

\section{Animal handling, tissue processing, and histopathology techniques}

All dogs were euthanized under pentothal deep anaesthesia, following xylazine sedation, and were transcardially perfused with normal saline followed by $4 \%$ paraformaldehyde in $0.1 \mathrm{M}$ phosphate buffer. Two age-matched dogs sacrificed during unrelated non-neurological diseases, were processed in parallel. Tissue samples were taken from the brain (brain stem and cerebellum), spinal cord (cervical, thoracic and lumbar segments), nerve dorsal roots (DR), dorsal root ganglia (DRG), and peripheral nerves both from fore- and hindlimbs. Median, ulnar and radial, sciatic, common peroneal, tibial, sural and musculocutaneous nerves were collected. Also, the vagus nerve, external and internal branches of the superior laryngeal nerve (SLN) and the recurrent laryngeal nerve (RLN) were taken and embedded in paraffin and routinely processed for $6 \mu \mathrm{m}$-thick sections. Dewaxed sections were stained with the following histological techniques: hematoxylin and eosin (H\&E), Luxol-fast blue-Klüver Barrera and Bielschowsky (BLS) or processed for immunohistochemistry (IHC) with appropriate primary antibodies. Masson's trichrome stain was performed in DRG and peripheral nerves to reveal possible fibrosis. In addition, small blocks of sciatic, sural, median, superior laryngeal nerve and RLN nerves were fixed in $2.5 \%$ glutaraldehyde, dehydrated through alcohol solutions, stained with $4 \%$ osmium tetroxide and embedded in Araldite. Semithin sections obtained were stained with toluidine blue and observed under the light microscope.

Histologically stained sections were examined by two independent researchers and evaluated for neuronal and axonal degeneration and loss, as revealed by BLS. Demyelination and other myelin abnormalities were evaluated in Luxol fast blue-Klüver Barrera paraffin sections or toluidine blue stained semithin sections. The spatial distribution of neuronal vacuolation was also investigated.

\section{Immunohistochemistry}

A set of IHC and immunofluorescence (IF) techniques were performed on paraffin sections to reveal astrocytic/microglia activation and neurotransmitter alterations in the cerebellum. Glutamate is the prevalent excitatory neurotransmitter for both the mossy fibre and climbing fibre system. Mossy fibres form synapses on granule cells in the GCL of the cortex and on neurons in the DCN. Climbing fibres coming from inferior olive nuclei form excitatory synapses directly on PCs and are strongly enriched in glutamate. In DCN, there are large glutamatergic neurons that project to premotor areas and directly regulate the motor control. On the other hand, GABA is the primary inhibitory neurotransmitter known to counterbalance the action of glutamate. PCs, the sole output neurons of the cerebellar cortex are GABAergic and project to the DCN and vestibular nuclei neurons. The DCN and vestibular nuclei are involved in motor control in animals through their communication with the nuclei of the thalamus and brainstem. GABA is also the predominant transmitter of cerebellar interneurons (basket cells, stellate cells, Golgi cells, Lugaro cells), except for unipolar brush cells, which are glutamatergic interneurons. In DCN, apart from the large glutamatergic neurons, there are small GABAergic projection neurons that send outputs to inferior olive nuclei and GABAergic local interneurons. The activities of GABA are mediated by vesicular or non-vesicular release after GABA is synthesized by glutamate decarboxylase (GAD), an enzyme that catalyses the decarboxylation of glutamate to GABA. The availability of antibodies against the neurotransmitters glutamate and GABA, and their receptors, such the $N$-methyl$D$-aspartate receptor1 (NMDAR1), which mediates neuronal functions in glutamate neurotransmission, 
Table I. Primary antibodies used

\begin{tabular}{|lcccc|}
\hline Antibody & & Supplier & Dilution & \multicolumn{1}{c|}{ Ad hoc References } \\
\hline Anti-GFAP & Rabbit polyclonal & $\begin{array}{c}\text { Dako Corporation, } \\
\text { Carpinteria, CA }\end{array}$ & $1: 1000$ & De Nevi et al. Eur J Histochem 2013; 57: e9 \\
\hline Anti-Iba1 & Rabbit polyclonal & Wako, Osaka, Japan & $1: 1000$ & $\begin{array}{l}\text { Lab Anim Res 2012; 28: 165-170 } \\
\text { Ahmed et al. J Histochem Cytochem 2007; } \\
\text { 5n: 687-700 }\end{array}$ \\
\hline Anti-GABA & Rabbit polyclonal & Sigma, St. Louis, USA & $1: 500$ & Am J Med Genet 1995; 57: 204-212 \\
\hline Anti-GAD67 & Mouse monoclonal & Millipore, Temecula, CA & $1: 1000$ & Neurosci Lett 2008; 431: 251-255 \\
\hline Anti-glutamate & Rabbit polyclonal & Millipore, Temecula, CA & $1: 500$ & $\begin{array}{c}\text { Am J Vet Res 2005; 66: 791-799 } \\
\text { J Vet Med Sci 2005; 67: 1119-1126 }\end{array}$ \\
\hline Anti-NMDAR1 & Mouse monoclonal & BD Pharmingen & $1: 250$ & Brain Res 1996; 723: 77-89 \\
\hline Anti-calbindin & Rabbit polyclonal & Millipore, Temecula, CA & $1: 800$ & Res Vet Sci 2010; 88: 122-126 \\
\hline
\end{tabular}

has made it possible to examine the neurohumoral synaptic transmission in the cerebellum and brain stem that are of interest in NVSD. Details of the antibodies used and their dilutions are given in Table I.

IHC was carried out using the avidin-biotin-peroxidase complex $(A B C)$ method. In brief, antigen retrieval was performed by incubating deparaffinized - rehydrated sections in citrate buffer $0.01 \mathrm{M}(\mathrm{pH} \mathrm{6)}$ under microwave treatment at 750 Watt for 3 minutes and thereafter at 350 Watt for 7 minutes. These sections were cooled down at RT for 20 minutes and thereafter were treated with $2 \%$ hydrogen peroxide in $5 \%$ methanol for 20 minutes followed by $5 \%$ normal goat serum for $2 \mathrm{~h}$, and subsequently one of the primary antibodies were applied overnight at $4^{\circ} \mathrm{C}$. Labelling was visualized with DAB (Vector) after pre-treatment with the $A B C$ kit (Vector, Vectastain) and sections were counterstained with haematoxylin. For IF the following secondary antibody: anti-mouse Alexa Fluor 488 and anti-rabbit Alexa fluor 488 (all in dilution 1 : 400, Molecular Probes) were used. Two observers, blinded to the identity of tissue sections, reviewed the IHC and IF preparations independently.

\section{Quantitative analysis \\ of neurotransmitter alterations and their receptors}

Seven to ten representative sections IHC stained for each antigenic marker were used for quantifications and photomicrographs were captured using a Nikon upright fluorescence microscope D-Eclipse 80i C1. Photomicrographs were subjected to digital optical densitometry with ImageJ, version $1.51 \mathrm{f}$ (National Institutes of Health, Bethesda, Maryland, USA), and were analysed by the following methods.
One method, which was used to quantify the intracellular GABA, NMDAR1 and calbindin integrated optical density (IOD) in neurons, integrates the grey value of the inverted image. For GAD, IF labelling was used and for the measurement of IOD there was no need to invert the images. The second method, which measures the area over which the immunoreactivity exceeds a given threshold, was applied to the glutamate and GABA staining for the quantification of glutamatergic and GABAergic fibres, respectively.

\section{Statistical analyses}

Statistical analyses were performed with non-parametric tests. Differences between two groups (NVSD vs. controls) per anatomical area were assessed with Wilcoxon Mann-Whitney $U$ tests. SPSS v20.0 statistical software system (IBM Corporation, Armonk, New York, USA) was used for calculations. The reported $p$ values were the result of two-tailed tests; $p$ values smaller or equal to 0.05 were considered statistically significant.

\section{Results \\ Lesions in peripheral nerves and the spinal cord}

Major histopathological lesions observed are summarized in Table II and have already been described by others. In relation to peripheral nerves, there was axonopathy with dystrophic axons, which was more severe in sensory nerves. In semithin sections, there was lack of large myelin sheaths and endoneurial fibrosis. RLN and SLN showed a prevalence of small-diameter fibres with axonal degeneration. Shrinkage of DRs with lack of large axons and mild fibrosis constitute the major abnormality of the DRG on NVSD. Vacuolation of 
Table II. Histopathological findings in NVSD-affected dogs

\begin{tabular}{|c|c|}
\hline Peripheral nerves & $\begin{array}{l}\text { 1. Loss of large-diameter myelinated fibres and prevalence of small-diameter fibres. } \\
\text { 2. Lack of large myelin sheaths. } \\
\text { 3. Thinning of axons and their myelin sheaths. } \\
\text { 4. Axonopathy with dystrophic axons. } \\
\text { 5. Endoneurial fibrosis. }\end{array}$ \\
\hline Dorsal spinal roots & $\begin{array}{l}\text { 1. Shrinkage. } \\
\text { 2. Lack of large axons. } \\
\text { 3. Predominance of small myelinated fibres. } \\
\text { 4. Mild fibrosis. }\end{array}$ \\
\hline Ventral spinal roots & Well preserved. \\
\hline Dorsal root ganglia & $\begin{array}{l}\text { 1. Increase of satellite cells (S-100+ and GFAP+ cells) } \\
\text { 2. Presence of residual nodes. } \\
\text { 3. Reactive fibrosis (proliferation of small spindle-shaped cells). } \\
\text { 4. Reduction of large pseudounipolar neurons. } \\
\text { 5. Presence of dystrophic axons. }\end{array}$ \\
\hline \multicolumn{2}{|l|}{ Spinal cord } \\
\hline White matter & $\begin{array}{l}\text { 1. Fasciculi mainly affected: gracile and cuneate fascicles, dorsal and lateral spinocerebellar, lateral } \\
\text { corticospinal fasciculi. } \\
\text { 2. Loss of large myelinated fibres. } \\
\text { 3. Axonal degeneration, with axons forming spheroids or ovoids. } \\
\text { 4. Myelin pallor. }\end{array}$ \\
\hline Grey matter & $\begin{array}{l}\text { 1. Shrinkage of dorsal horns. } \\
\text { 2. Neuronal loss in Clarke's column. } \\
\text { 3. Vacuolated interneurons in laminae VI and VII. }\end{array}$ \\
\hline \multicolumn{2}{|l|}{ Brain stem } \\
\hline Vestibular nuclei & $\begin{array}{l}\text { 1. Neuronal vacuolation in the following nuclei: 1) dorsal column nuclei (gracilis and cuneatus), } \\
\text { 2) spinal trigeminal nucleus, 3) Deiter's nucleus (nD) or nucleus vestibularis inferior, 4) triangular } \\
\text { nucleus or nucleus vestibularis medialis, 5) nucleus of Roller (nR) or sublingual nucleus and } \\
\text { 6) nucleus of the solitary tract. } \\
\text { 2. Microglia activation and mild astrocytosis. }\end{array}$ \\
\hline White matter & Axonal degeneration and myelin pallor. \\
\hline Olivary nuclei & $\begin{array}{l}\text { 1. Vacuolated neurons. } \\
\text { 2. Partially spongiform appearance of the structure. } \\
\text { 3. Atrophy of large neurons and diffuse axonal loss. }\end{array}$ \\
\hline \multicolumn{2}{|l|}{ Cerebellum } \\
\hline Cerebellar nuclei & $\begin{array}{l}\text { 1. Profound neuronal vacuolation. } \\
\text { 2. Loss of large neurons. } \\
\text { 3. Atrophy of cerebellar nuclei and their efferent myelinated fibres. } \\
\text { 4. Astrocytosis and focal microgliosis. } \\
\text { 5. Spongiform appearance. } \\
\text { 6. Myelin pallor and axonal loss in neuropils. }\end{array}$ \\
\hline \multirow[t]{3}{*}{ Cerebellar cortex } & Altered fragmented dendritic trees of Purkinje cells. \\
\hline & $\begin{array}{l}\text { Atrophy and loss in most cerebellar lobules. } \\
\text { Mild Bergmann gliosis. }\end{array}$ \\
\hline & Axonal torpedoes belonging to the axons of Purkinje cells. \\
\hline
\end{tabular}

pseudounipolar neurons of DRG was not observed, but moderate neuronal loss of large neurons was evident. Dystrophic axons characterized by focal dilations and bulb like structures were also noted.

In the white matter of SC, lesions were mainly located in gracile and cuneate fascicles, in dorsal spinocerebellar and lateral corticospinal fasciculi. In these areas, myelin sheaths were found either empty or occasionally containing swollen axons forming spheroids or ovoids. In cervical SC, the axonal degeneration and myelin loss were most prominent in the lateral spinocerebellar tract. In spinal grey matter, 
the most profound finding was the shrinkage of the dorsal horn and its neuronal loss, most evident in Clarke's column. Vacuolated neurons were found mainly in the medial part of laminae VI and VII presumably representing interneurons and observed sparsely only in the ventral horn of SC.

\section{Neuropathology of the brain stem}

In the brain stem, there was a moderate vacuolation of neurons bilaterally in many nuclei of the dorsal medulla oblongata (Table II and Fig. 1). Neuronal vacuolation was characterized by intracytoplasmic single or multiple vacuoles of variable size, ranging from 3 to $40 \mu \mathrm{m}$, arranged either individually or in clusters. Vacuoles were round and empty and in paraffin-embedded sections, were not stained with histochemical methods (Figs. 2A and B). Neuronal vacuolation was accompanied by microglia activation and mild astrocytosis. In the inferior olivary nucleus, the presence of vacuolated neurons resulted in the spongiform appearance of the structure. Atrophy of large neurons in the olivary nucleus and diffuse axonal loss were also evident. IHC showed that the median IOD of GAD in the remaining neurons of the olivary nucleus of NVSD animals was elevated approximately twofold compared with controls and this difference was statistically significant. Medians of GAD IODs in NVSD and controls were 52,023 and 18,556, respectively; the distributions in the two groups differed significantly (Mann-Whitney $U=11.5, z=-5.307, p<0.001$ two-tailed, Fig. 4C).

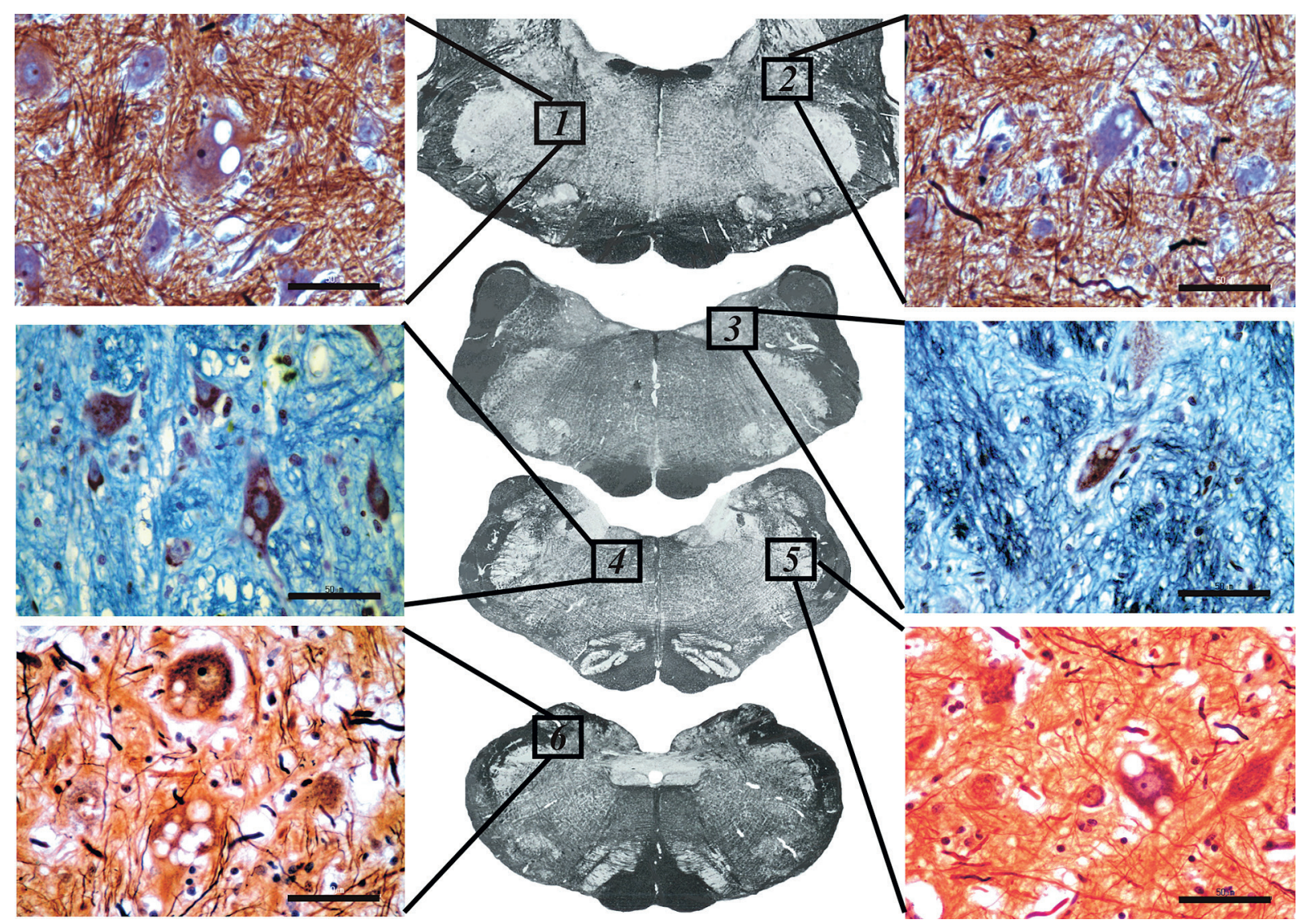

Fig. 1. Photomicrographs of transverse sections through different segments of the brain stem in NVSD animals stained with either BLS or Luxol Fast blue-Klüver Barrera. Vacuolated neurons were detected mainly at: 1 - the reticular formation, 2 - the descent vestibular nucleus, 3 - the medial vestibular nucleus, 4 - the nucleus of Roller, 5 - the nucleus of the trigeminal nerve, 6 - the external nucleus of fasciculus cuneatus. Scale bars are $50 \mu \mathrm{m}$. 

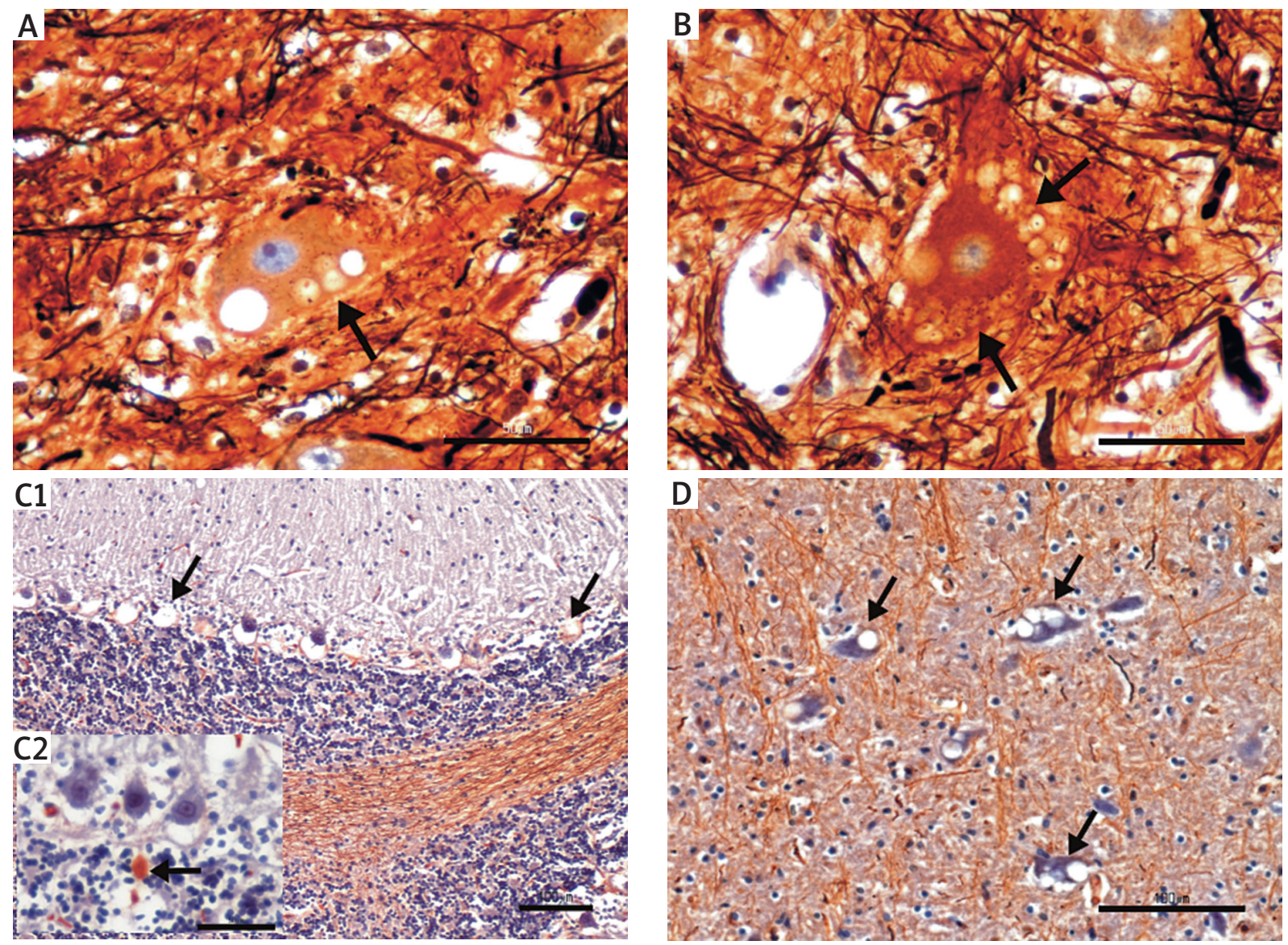

Fig. 2. Representative photomicrographs of cerebellum paraffin sections stained with BLS summarizing the neuropathology in NVSD animals. A, B) Neuronal vacuolation was characterized by single or multiple vacuoles (of variable size) and was more profound in cerebellar nuclei. Scale bars in A, B are $50 \mu \mathrm{m}$. C1-2,D) In the cerebellar cortex (C1), the atrophy and loss of Purkinje cells were evident leaving empty baskets (arrows). Axonal torpedoes in the granular cell layer belonging to degenerative Purkinje cells were also detected (arrow in C2). In cerebellar nuclei (D), the neuronal vacuolation (arrows) and axonal loss were profound, resulting in spongiform appearance of the parenchyma. Scale bars in C, D are $50 \mu \mathrm{m}$.

\section{Neuropathology of the cerebellum}

Loss of the large (glutamatergic) neurons and neuronal vacuolation of the remaining cells constitute the most striking histopathological lesions seen in the DCN of NVSD animals. DCN neurons showed single or multiple sharply demarcated cytoplasmic vacuoles which sometimes resulted in peripheral margination of the nucleus (Fig. 2D). In NVSD animals, IOD of GAD in the remaining neurons either vacuolated or not, significantly declined (medians of GAD IOD in NVSD and controls were 16,189 and 128,583; the distributions in the two groups differed significantly (Mann-Whitney $U=0.00$, $z=-5.477, p<0.001$, two-tailed, Figs. 3A, B and 4C).
Similarly, the median IOD of GABA in NVSD animals was reduced by $45 \%$ compared to controls. Medians of GABA IOD in NVSD and controls were $19.17 \times 10^{6}$ and $34.75 \times 10^{6}$, respectively; the distributions in the two groups differed significantly (Mann-Whitney $U=24.00, z=-2.225, p=0.024$, two-tailed, Figs. 3C, $D$ and $4 F)$. In contrast, the median IOD of NMDAR1 in DCN neurons of NVSD animals was elevated by $47 \%$ compared with control animals and this difference reached statistical significance (medians were $49.08 \times 10^{6}$ and $33.41 \times 10^{6}$, respectively; MannWhitney $U=27.00, z=-2.2, p=0.028$, two-tailed, Figs. 3F, G and H). DCN neurons were positive for calbindin, although variations on staining inten- 

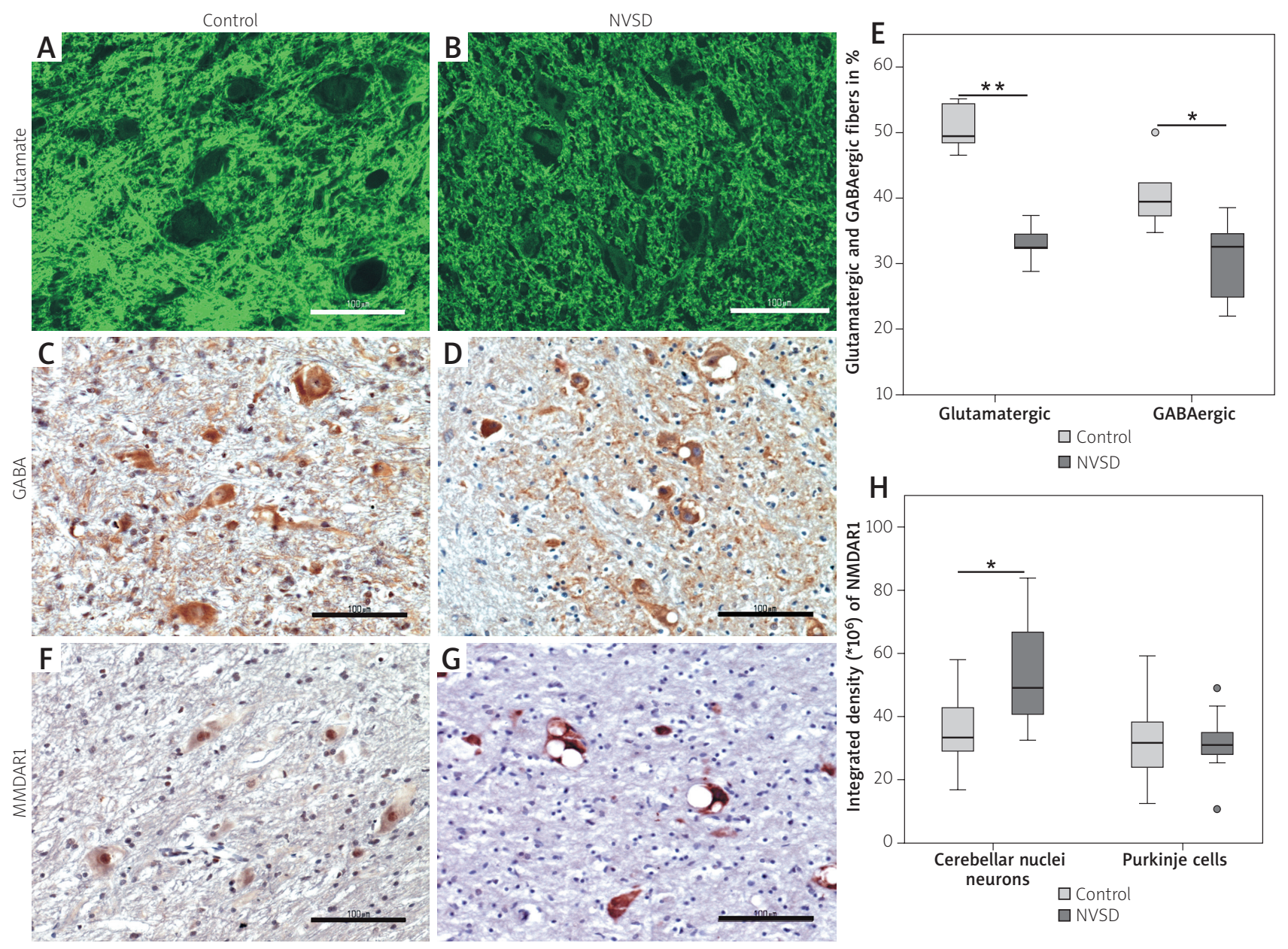

Fig. 3. A-D, F, G) Photomicrographs of paraffin sections of the cerebellum depicting various areas of cerebellar nuclei in control and NVSD animals immunohistochemically stained for glutamate (A, B), GABA (C, D) and $\operatorname{NMDAR1}(\mathrm{F}, \mathrm{G})$. A, B) IHC for glutamate revealed a reduction in glutamatergic fibres, which is expressed as a reduction in the pixel area \% per optical field in graph E. C, D) Apart from the reduction in IOD of GABA in neuronal somata, there was also a significant reduction in the GABAergic fibres in cerebellar nuclei of NVSD animals. F, G) Cerebellar nuclei neurons exhibited a strong immunoreactivity for NMDAR1 in NVSD animals $\left({ }^{*} p<0.05\right)$. Scale bars are $100 \mu \mathrm{m}$. E) Graph illustrating the percentage of the pixel area of glutamatergic and GABAergic fibres per optical field in cerebellar nuclei in control and NVSD animals. The boxplots represent the median value ( $50^{\text {th }}$ percentile) and the range of $\%$ area of immunoreactive fibres. The outliers (values that are $>1.5$ the interquartile range [IQRs]) are marked with a circle. There was a significant reduction in glutamatergic and GABAergic fibres in NVSD animals ( $\left.{ }^{* *} p<0.01,{ }^{*} p<0.05\right)$. H) Graph illustrating the integrated optical density (IOD) of NMDAR1 in cerebellar nuclei neurons and Purkinje cells in control and NVSD animals. The boxplots represent the median value ( $50^{\text {th }}$ percentile) and the range of IOD. The IOD of NMDAR1 was significantly higher in cerebellar nuclei neurons of NVSD animals compared to controls ( $\left.{ }^{*} p<0.05\right)$.

sity were noted. The cytoplasm of DCN neurons showed a severe decrease in IOD of calbindin compared to controls (medians were $8.74 \times 10^{6}$ and $45.65 \times 10^{6}$, respectively; Mann-Whitney $U=$ $0.00, z=-3.182, p=0.001$, two-tailed, Fig. 4l), suggesting an altered $\mathrm{Ca}^{+}$dependent metabolism.
In parallel with the vacuolation, the applied histological techniques revealed a spongiform change, myelin pallor and axonal loss within the neuropil. Astrocytosis and focal microgliosis were confirmed by GFAP and Iba1 IHC, respectively. In DCN there was a $24.4 \%$ loss of GABAergic fibres in NVSD compared 



Fig. 4. A, B, D, E, G, H) Photomicrographs of paraffin sections of the cerebellum depicting the cerebellar cortex in control and NVSD animals immunohistochemically stained for GAD (A, B), GABA (D, E) and calbindin (G, H). A, B) IHC for GAD revealed a reduction in immunoreactivity of GAD in Purkinje cells and elimination of GAD positive terminals in the granular cell layer (GCL) of NVSD animals (arrowheads in A indicate the GAD positive terminals in GCL). "Empty baskets" (arrows) in B indicate the sites of atrophic Purkinje cells. Atrophy of Purkinje cells was accompanied by a decrease in immunoreactivity of GABA (E) and calbindin (H) in NVSD animals. Calbindin IHC revealed the characteristic dendritic trees of Purkinje cells (arrowheads in $\mathbf{G}$ ) in the molecular cell layer (MCL) of control animals. Scale bars are $100 \mu \mathrm{m}$. C, F, I) Graphs illustrating the integrated optical density (IOD) of GAD, GABA and calbindin respectively, in cerebellar nuclei neurons and Purkinje cells (and in olive neurons for GAD) in control and NVSD animals. The boxplots represent the median value $\left(50^{\text {th }}\right.$ percentile) and the range of IOD. The outliers (values that are $>1.5$ the interquartile range [IQRs]) are marked with a circle. The IODs of GAD, GABA and calbindin were significantly lower $\left({ }^{*} p<0.05,{ }^{* *} p<0.01\right.$, $\left.{ }^{* * *} p<0.001\right)$ in cerebellar nuclei neurons and in Purkinje cells of NVSD animals compared to controls.

with control animals and this difference reached statistical significance (medians were $32.563 \%$ and $39.446 \%$, respectively; Mann-Whitney $U=2.00, z=$ $-2.373, p=0.018$, two-tailed, Figs. 3C-E) indicative of the impaired cerebellar corticonuclear connections. In parallel, there was a severe reduction (32\%) in gluta- matergic fibres in NVSD animals (32.361\% vs. 49.431\%; Mann-Whitney $U=0.00, z=-2.611, p=0.009$, twotailed, Figs. 3A, B and E), suggesting the decline of glutamatergic inputs in DCN.

In the cerebellar cortex, atrophy and irregular loss of PCs were prominent in all dogs, accompa- 
nied by mild Bergmann gliosis and "empty baskets" in the cerebellar cortex (Fig. 2 C1). The GCL exhibited shrinkage and BLS staining revealed occasional axonal torpedoes in this layer belonging to the axons of the PCs (Fig. 2 C2). The median IOD of GAD in PCs of NVSD animals significantly declined compared to controls. Medians of GAD IODs were 36,324 and 104,648 respectively; the distributions in the two groups differed significantly (Mann-Whitney $U=$ 2.00, $z=-2.817, p=0.005$, two-tailed, Figs. 4A-C). In PCS IOD of GABA severely reduced in NVSD compared to control animals. Medians of GABA IODs were $26.70 \times 10^{6}$, and $61.64 \times 10^{6}$, respectively; the distributions in the two groups differed significantly (Mann-Whitney $U=7.00, z=-3.207, p=0.001$, twotailed, Figs. 4D-F). Similarly, IHC for calbindin, revealed a significant reduction in IOD of PCS in NVSD compared to controls (medians were $28.23 \times 10^{6}$ and $62.60 \times 10^{6}$, respectively; Mann-Whitney $U=0.00$, $z=-3.919, p<0.001$, two-tailed) and altered fragmented dendritic trees in the molecular cell layer (Figs. 4G-I). IOD of NMDAR1 by PCs did not differ significantly between NVSD and control animals (medians were $30.97 \times 10^{6}$ and $31.49 \times 10^{6}$, respectively; Mann-Whitney $U=73.20, z=-0.189, p=0.85$, twotailed, Fig. 3H).

\section{Discussion}

Recently the RAB3GAP1:c.743delC variant has been identified in the homozygous state in all NVSDaffected Rottweilers. None of the normal Rottweilers or the Rottweilers with signs beginning at $>1$ year of age were homozygous for the variant. The RAB3GAP1:C.743delC produces a frame shift that predicts a premature stop codon and a truncated gene product RAB3GAP1:p.P248Lfs4, missing 730 C-terminal amino acids, including the catalytic domain, therefore it is doubtful that the truncated gene product retains biological activity [32].

RAB3GAP1 codes for the catalytic subunit that combines with a non-catalytic subunit encoded by RAB3GAP2 to form Rab3GAP. Rab3GAP was first recognized as a GTPase activator protein (GAP) that greatly enhances the inherent GTPase activity of Rab3 [14,23,37]. Rab proteins function as molecular switches that regulate the formation, transport, tethering and fusion of a variety of membrane structures by cycling between inactive GDP-bound and active GTP-bound states $[8,23]$. These proteins play a vital role in membranous transport within the cell, allowing movement of cell organelles, and endocytosis and exocytosis [11,17,46] reviewed in [40,53]. The mechanism by which they achieve this has been well characterized for certain members of the family, and depends on their GTP state, and their ability to prenylate and thus cycle on and off membranes [1]. However, the precise point of action of Rab3 GAP in synaptic vesicle transport has not been clear so far.

It has been found that in Rab3 GAP p130-deficient mice, the GTP-bound form of Rab3A accumulates in the brain and the $\mathrm{Ca}^{2+}$-dependent glutamate release from cerebrocortical synaptosomes is inhibited [42]. This observation suggests that Rab3 GAP inactivates Rab3A in nerve terminals and thereby regulates neurotransmitter release and synaptic plasticity $[23,34,47]$. Consistent with this prediction, mutations in 130 have recently been reported to cause WARBM and the altered neurotransmission could be involved in the pathogenesis of this syndrome [2,3]. Gerondopoulos et al. [16] reported that Rab3GAP not only functions as a GAP for the Rab3s but also functions as a guanine exchange factor (GEF) for Rab18. Supporting the view that Rab18 activity is important for ER structure, in the absence of either Rab3GAP subunit or Rab18 function, ER tubular networks were disrupted, and ER sheets spread out into the cell periphery [16]. It has been suggested that the intra-neuronal vacuoles may be microscopic and ultrastructural manifestations of a malfunctioned ER that has been aberrantly distributed due to the absence of Rab3GAP activity [32].

A predominant clinical feature of the NVSD phenotype is the juvenile onset laryngeal paralysis and polyneuropathy due to axonopathy. Dystrophic changes in nerves of NVSD-affected dogs have been extensively studied and confirmed by others [7,9] and are characterized by accumulations of normal or abnormal appearing organelles and clusters of vesicular structures resembling proliferating $E R$, arising from an altered axoplasmic flow [29]. Dystrophic changes were reported in axons of the sciatic nerve in Rab18 knockout mice with disorganization of neurofilaments and collections of microtubules predominantly at the neuromuscular junctions [10]. WARBM can be caused directly by loss of RAB18, or indirectly through loss of RAB18 regulators, RAB3GAP or TBC1D20 [6,19]. In Rab18 knockout mice, neurons with longer axons are more severely affected than those with shorter axons. Interestingly, also in NVSD 
the thick myelinated axons are more severely affected. Finding such changes remote from the cell body suggests that axonal transport of membranes and/ or vesicles is disrupted by the RAB3GAP1 mutation. Moreover, it is tempting to speculate that an anterograde trans-synaptic degeneration is involved in the pathogenesis of neuronal vacuolation.

The link between axonopathy and neuronal vacuolar degeneration at distinctive anatomical pathways has been reported in several animal models at both spontaneous and experimental lesions [21,36,50]. Neurons within some nuclei in the CNS can atrophy and degenerate via vacuolation in response to deafferentation. Examples are neurons in the inferior and accessory olivary nuclei, which undergo an unusual form of trans-synaptic degeneration after a destructive lesion of the ipsilateral central tegmental tract. The olivary ribbon becomes thickened and neurons show marked enlargement, cytoplasmic vacuolation, and some dispersion of Nissl bodies [48]. In NVSD, large vacuoles were visible mainly within neurons of the DCN, vestibular nuclei and inferior olive. In other areas in the CNS, neuronal vacuolation was either absent or limited, indicating a selective susceptibility of specific neuronal types to vacuolation. Overall, vacuolation occurred in interconnected extrapyramidal and oculomotor structures and this spatial pattern of distribution suggests the spread of this disorder across synaptic connections [44]. However, we cannot exclude the possibility that any abnormalities in other neurons are not gross, but might be subtler at the subcellular level.

Many studies have addressed the selective vulnerability of DCN neurons to NVSD $[13,15,27,41,43]$, however none of them examined either the subtypes of neurons mainly affected or the relative importance of neurohumoral synaptic dysfunction in this structure. The normal DCN contains mainly three populations of neurons: small GABAergic neurons, which send projections to the inferior olive, small GABAergic local interneurons and larger glutamatergic neurons, which project to premotor areas and regulate the motor control [22]. These cells are GAD positive with a mild expression of GABA and NMDAR1. In NVSD cerebellar nuclei, large neurons are severely in decline in parallel with a reduction in GABA and GAD immunoreactivity of the remaining cells.

Moreover, in normal DCN, nuclear neurons receive mostly inhibitory GABAergic inputs from PCs and excitatory glutamatergic inputs from mossy fibres.
The latter originate from the second order neuron of Clarke's nuclei and from axon collaterals of inferior olive neurons. In NVSD animals, the glutamatergic inputs in DCN severely declined in parallel with the overexpression of NMDAR1 by the neuronal somata. This upregulation probably serves as a compensatory mechanism in the reduction of excitatory glutamatergic inputs from mossy fibres. However, GABAergic synapses formed by the axons of PCs outnumber the glutamatergic and GABAergic synapses formed by local interneurons [51]. The reduction of GABAergic fibres in DCN in NVSD animals is substantial suggesting that GABAergic inhibition from PCs is heavily missing.

Intraneuronal vacuoles with a similar distribution pattern as in NVSD have been recently reported in a spontaneous neurodegenerative disease in Lagotto Romagnolo dogs associated with abnormal autophagosome maturation and a mutation in the autophagy-related gene ATG4D [28]. There are a variety of studies, either in transgenic mice or canine hereditary ataxias providing compelling evidence that a primary defect in autophagy can induce neurodegenerative disease. Autophagy is the process by which more long-lived proteins and organelles are incorporated into autophagosomes for delivery to vacuoles or lysosomes for degradation. Constitutive autophagy plays an important role as a basal source of energy in cells with high metabolic needs, such as the PCs, in the global turnover of cellular organelles and in the clearance of potentially toxic protein aggregates [2426]. Is it well acknowledged that the heterodimeric RAB3GAP complex regulates the RAB GTPase RAB3 and the release of neurotransmitters at the neuronal synapse; however, recently it has been found that RAB3GAP1/2 also affects intracellular protein aggregation and modulate autophagosomal maturation in basal and rapamycin-induced conditions [45]. Deficiencies of glutamatergic and GABAergic terminals in the DCN of NVSD animals may induce a compromised function of proteins involved in the organelles' integrity. Moreover, vacuolation in DCN neurons may provide evidence for an altered autophagy as a stress response to anterograde transneuronal deafferentation and neurotransmitters deprivation [35].

Atrophy and loss of PCs constitute a major histopathological hallmark in the cerebellum of NVSD animals. The remaining PCs showed reduced IOD for GAD, GABA and calbindin. In addition, histopathology revealed axonal torpedoes, which are 
ellipsoid swellings of PC axons in the GCL and are considered to be non-specific changes in various disorders, such as spinocerebellar degeneration [31]. Torpedo formation has been linked to the loss of DCN neurons as a consequence of a retrograde change based on the synaptic detachment of PC axon terminals. It is unlikely that primary changes in PC bodies are involved in the torpedo formation seen in these conditions. On the contrary, the fact that there is a severe damage of DCN but a number of PCs is preserved raises the possibility that the neuronal loss leads secondarily to torpedo formation. Moreover, it has been shown that torpedoes do not develop until the density of neurons in DCN is reduced to about half of that in controls, because the terminal arborisation of the axon of each PC occurs in the DCN to make synaptic contact with multiple neurons [31].

In conclusion, vacuolation in diseases such as NVSD could be a histopathological manifestation of intense intracellular degradation of specific neuronal types, due to neurotransmitters deprivation in the terminals of afferent fibres. The RAB3GAP1:c.743delC dogs could serve as a model for investigating the role of Rab3GAP in membrane trafficking and the pathogenesis of diseases such as WARBM.

\section{Acknowledgements}

Dr Polyzopoulou Zoi is thanked for the generous donation of affected dogs, Dr Tsingotjidou Anastasia and Dr Bekiari Chryssa for their valuable contribution, Chiotelli Maria for her excellent technical assistance, and Dr Papadopoulos GC for his constructive criticism on the original manuscript. The study was supported by National Funds.

\section{Disclosure}

The author reports no conflict of interest.

\section{References}

1. Agler C, Nielsen DM, Urkasemsin G, Singleton A, Tonomura N, Sigurdsson S, Tang R, Linder K, Arepalli S, Hernandez D, Lindblad-Toh K, van de Leemput J, Motsinger-Reif A, O'Brien DP, Bell J, Harris T, Steinberg S, Olby NJ. Canine hereditary ataxia in old english sheepdogs and gordon setters is associated with a defect in the autophagy gene encoding RAB24. PLoS Genet 2014; 10: e1003991.

2. Aligianis IA, Johnson CA, Gissen P, Chen D, Hampshire D, Hoffmann K, Maina EN, Morgan NV, Tee L, Morton J, Ainsworth JR,
Horn D, Rosser E, Cole TR, Stolte-Dijkstra I, Fieggen K, ClaytonSmith J, Megarbane A, Shield JP, Newbury-Ecob R, Dobyns WB, Graham JM, Jr., Kjaer KW, Warburg M, Bond J, Trembath RC, Harris LW, Takai Y, Mundlos S, Tannahill D, Woods CG, Maher ER. Mutations of the catalytic subunit of RAB3GAP cause Warburg Micro syndrome. Nat Genet 2005; 37: 221-223.

3. Aligianis IA, Morgan NV, Mione M, Johnson CA, Rosser E, Hennekam RC, Adams G, Trembath RC, Pilz DT, Stoodley N, Moore AT, Wilson S, Maher ER. Mutation in Rab3 GTPase-activating protein (RAB3GAP) noncatalytic subunit in a kindred with Martsolf syndrome. Am J Hum Genet 2006; 78: 702-707.

4. Armstrong RA. Can neurodegenerative disease be defined by four 'primary determinants': anatomy, cells, molecules, and morphology? Folia Neuropathol 2016; 54: 89-104.

5. Armstrong RA, Cairns NJ, Lantos PL. Are pathological lesions in neurodegenerative disorders the cause or the effect of the degeneration? Neuropathology 2002; 22: 133-146.

6. Bem D, Yoshimura S, Nunes-Bastos R, Bond FC, Kurian MA, Rahman F, Handley MT, Hadzhiev Y, Masood I, StraatmanIwanowska AA, Cullinane AR, McNeill A, Pasha SS, Kirby GA, Foster K, Ahmed Z, Morton JE, Williams D, Graham JM, Dobyns WB, Burglen L, Ainsworth JR, Gissen P, Muller F, Maher ER, Barr FA, Aligianis IA. Loss-of-function mutations in RAB18 cause Warburg micro syndrome. Am J Hum Genet 2011; 88: 499-507.

7. Bennett PF, Clarke RE. Laryngeal paralysis in a rottweiler with neuroaxonal dystrophy. Aust Vet J 1997; 75: 784-786.

8. Bhuin T, Roy JK. Rab proteins: the key regulators of intracellular vesicle transport. Exp Cell Res 2012; 328: 1-19.

9. Braund KG, Steinberg HS, Shores A, Steiss JE, Mehta JR, ToivioKinnucan M, Amling KA. Laryngeal paralysis in immature and mature dogs as one sign of a more diffuse polyneuropathy. J Am Vet Med Assoc 1989; 194: 1735-1740.

10. Carpanini SM, McKie L, Thomson D, Wright AK, Gordon SL, Roche SL, Handley MT, Morrison H, Brownstein D, Wishart TM, Cousin MA, Gillingwater TH, Aligianis IA, Jackson IJ. A novel mouse model of Warburg Micro syndrome reveals roles for RAB18 in eye development and organisation of the neuronal cytoskeleton. Dis Model Mech 2014; 7: 711-722.

11. Corbeel L, Freson K. Rab proteins and Rab-associated proteins: major actors in the mechanism of protein-trafficking disorders. Eur J Pediatr 2008; 167: 723-729.

12. de Lahunta A, Summers BA. The laryngeal lesion in young dogs with neuronal vacuolation and spinocerebellar degeneration. Vet Pathol 1998; 35: 316-317.

13. Eger CE, Huxtable CR, Chester ZC, Summers BA. Progressive tetraparesis and laryngeal paralysis in a young rottweiler with neuronal vacuolation and axonal degeneration: an Australian case. Australian Veterinary Journal 1998; 76: 733-737.

14. Fukui K, Sasaki T, Imazumi K, Matsuura Y, Nakanishi H, Takai Y. Isolation and characterization of a GTPase activating protein specific for the Rab3 subfamily of small G proteins. J Biol Chem 1997; 272: 4655-4658.

15. Geiger DA, Miller AD, Cutter-Schatzberg K, Shelton GD, de Lahunta A, Schatzberg S.. Encephalomyelopathy and polyneuropathy associated with neuronal vacuolation in two Boxer littermates. Veterinary Pathology 2009; 46: 1160-1165. 
16. Gerondopoulos A, Bastos RN, Yoshimura S, Anderson R, Carpanini S, Aligianis I, Handley MT, Barr FA. Rab18 and a Rab18 GEF complex are required for normal ER structure. J Cell Biol 2014; 205: 707-720.

17. Goody RS, Rak A, Alexandrov K. The structural and mechanistic basis for recycling of Rab proteins between membrane compartments. Cell Mol Life Sci 2005; 62: 1657-1670.

18. Graham JM Jr., Hennekam R, Dobyns WB, Roeder E, Busch D. MICRO syndrome: an entity distinct from COFS syndrome. Am J Med Genet A 2004; 128A: 235-245.

19. Handley MT, Carpanini SM, Mali GR, Sidjanin DJ, Aligianis IA, Jackson IJ, FitzPatrick DR. Warburg Micro syndrome is caused by RAB18 deficiency or dysregulation. Open Biol 2015; 5: 150047.

20. Handley MT, Morris-Rosendahl DJ, Brown S, Macdonald F, Hardy C, Bem D, Carpanini SM, Borck G, Martorell L, Izzi C, Faravelli F, Accorsi P, Pinelli L, Basel-Vanagaite L, Peretz G, Abdel-Salam GM, Zaki MS, Jansen A, Mowat D, Glass I, Stewart H, Mancini G, Lederer D, Roscioli T, Giuliano F, Plomp AS, Rolfs A, Graham JM, Seemanova E, Poo P, Garcia-Cazorla A, Edery P, Jackson IJ, Maher ER, Aligianis IA. Mutation spectrum in RAB3GAP1, RAB3GAP2, and RAB18 and genotype-phenotype correlations in warburg micro syndrome and Martsolf syndrome. Hum Mutat 2013; 34: 686-696.

21. He L, Lu XY, Jolly AF, Eldridge AG, Watson SJ, Jackson PK, Barsh GS, Gunn TM. Spongiform degeneration in mahoganoid mutant mice. Science 2003; 299: 710-712.

22. Hirano T, Kawaguchi SY. Regulation of inhibitory synaptic plas ticity in a Purkinje neuron. Cerebellum 2012; 11: 453-454.

23. Hutagalung AH, Novick PJ. Role of Rab GTPases in membrane traffic and cell physiology. Physiol Rev 2011; 91: 119-149.

24. Komatsu M, Kominami E, Tanaka K. Autophagy and neurodegeneration. Autophagy 2006; 2: 315-317.

25. Komatsu M, Ueno T, Waguri S, Uchiyama Y, Kominami E, Tanaka K. Constitutive autophagy: vital role in clearance of unfavorable proteins in neurons. Cell Death Differ 2007; 14: 887-894.

26. Komatsu M, Waguri S, Chiba T, Murata S, Iwata J, Tanida I, Ueno T, Koike M, Uchiyama Y, Kominami E, Tanaka K. Loss of autophagy in the central nervous system causes neurodegeneration in mice. Nature 2006; 441: 880-884.

27. Kortz GD, Meier WA, Higgins RJ, French RA, McKiernan BC, Fatzer R, Zachary JF. Neuronal vacuolation and spinocerebellar degeneration in young Rottweiler dogs. Veterinary Pathology 1997; 34: 296-302.

28. Kyostila K, Syrja P, Jagannathan V, Chandrasekar G, Jokinen TS, Seppala EH, Becker D, Drogemuller M, Dietschi E, Drogemuller C, Lang J, Steffen F, Rohdin C, Jaderlund KH, Lappalainen AK, Hahn K, Wohlsein P, Baumgartner W, Henke D, Oevermann A, Kere J, Lohi $\mathrm{H}$, Leeb T. A missense change in the ATG4D gene links aberrant autophagy to a neurodegenerative vacuolar storage disease. PLoS Genet 2015; 11: e1005169.

29. Lampert PW. A comparative electron microscopic study of reactive, degenerating, regenerating, and dystrophic axons. J Neuropathol Exp Neurol 1967; 26: 345-368.

30. Mahony OM, Knowles KE, Braund KG, Averill DR Jr., Frimberger AE. Laryngeal paralysis-polyneuropathy complex in young Rottweilers. J Vet Intern Med 1998; 12: 330-337.
31. Matsumoto R, Nakano I, Arai N, Oda M, Yagishita S, Hashizume Y. Loss of the dentate nucleus neurons is associated with torpedo formation: a morphometric study in progressive supranuclear palsy and dentatorubro-pallidoluysian atrophy. Acta Neuropathol 1998; 95: 149-153.

32. Mhlanga-Mutangadura T, Johnson GS, Ashwini A, Shelton GD, Wennogle SA, Johnson GC, Kuroki K, O’Brien DP. A Homozygous RAB3GAP1:c.743delC Mutation in Rottweilers with Neuronal Vacuolation and Spinocerebellar Degeneration. J Vet Intern Med 2016; 30: 813-818.

33. Morris-Rosendahl DJ, Segel R, Born AP, Conrad C, Loeys B, Brooks SS, Muller L, Zeschnigk C, Botti C, Rabinowitz R, Uyanik G, Crocq MA, Kraus U, Degen I, Faes F. New RAB3GAP1 mutations in patients with Warburg Micro Syndrome from different ethnic backgrounds and a possible founder effect in the Danish. Eur J Hum Genet 2010; 18: 1100-1106.

34. Muller M, Pym EC, Tong A, Davis GW. Rab3-GAP controls the progression of synaptic homeostasis at a late stage of vesicle release. Neuron 2011; 69: 749-762.

35. Murrow L, Debnath J. Autophagy as a stress-response and quality-control mechanism: implications for cell injury and human disease. Annu Rev Pathol 2013; 8: 105-137.

36. Nagahama M, Suzuki M, Hamada Y, Hatsuzawa K, Tani K, Yamamoto A, Tagaya M. SVIP is a novel VCP/p97-interacting protein whose expression causes cell vacuolation. Mol Biol Cell 2003; 14: 262-273.

37. Nagano F, Sasaki T, Fukui K, Asakura T, Imazumi K, Takai Y. Molecular cloning and characterization of the noncatalytic subunit of the Rab3 subfamily-specific GTPase-activating protein. J Biol Chem 1998; 273: 24781-24785.

38. Nassogne MC, Henrot B, Saint-Martin C, Kadhim H, Dobyns WB, Sebire G. Polymicrogyria and motor neuropathy in Micro syndrome. Neuropediatrics 2000; 31: 218-221.

39. Oishi H, Sasaki T, Nagano F, Ikeda W, Ohya T, Wada M, Ide N, Nakanishi $H$, Takai Y. Localization of the Rab3 small G protein regulators in nerve terminals and their involvement in Ca2+-dependent exocytosis. J Biol Chem 1998; 273: 34580-14585.

40. Pereira-Leal JB, Seabra MC. The mammalian Rab family of small GTPases: definition of family and subfamily sequence motifs suggests a mechanism for functional specificity in the Ras superfamily. J Mol Biol 2000; 301: 1077-1087.

41. Pumarola M, Fondevila D, Borras D, Majo N, Ferrer I. Neuronal vacuolation in young Rottweiler dogs. Acta Neuropathologica 1999; 97: 192-195.

42. Sakane A, Manabe S, Ishizaki H, Tanaka-Okamoto M, Kiyokage E, Toida K, Yoshida T, Miyoshi J, Kamiya H, Takai Y, Sasaki T. Rab3 GTPase-activating protein regulates synaptic transmission and plasticity through the inactivation of Rab3. Proc Natl Acad Sci U S A 2006; 103: 10029-10034.

43. Salvadori C, Tartarelli CL, Baroni M, Mizisin A, Cantile C. Peripheral nerve pathology in two rottweilers with neuronal vacuolation and spinocerebellar degeneration. Veterinary Pathology 2005; 42: 852-855.

44. Saper CB, Wainer BH, German DC. Axonal and transneuronal transport in the transmission of neurological disease: potential role in system degenerations, including Alzheimer's disease. Neuroscience 1987; 23: 389-398. 
45. Spang N, Feldmann A, Huesmann H, Bekbulat F, Schmitt V, Hiebel C, Koziollek-Drechsler I, Clement AM, Moosmann B, Jung J, Behrends C, Dikic I, Kern A, Behl C. RAB3GAP1 and RAB3GAP2 modulate basal and rapamycin-induced autophagy. Autophagy 2014; 10: 2297-2309.

46. Stenmark H. Rab GTPases as coordinators of vesicle traffic. Nat Rev Mol Cell Biol 2009; 10: 513-525.

47. Sudhof TC. The synaptic vesicle cycle. Annu Rev Neurosci 2004; 27: 509-547.

48. Takamine K, Okamoto K, Fujita Y, Sakurai A, Takatama M, Gonatas NK. The involvement of the neuronal Golgi apparatus and trans-Golgi network in the human olivary hypertrophy. J Neurol Sci 2000; 182: 45-50.

49. Tiemeyer MJ, Singer HS, Troncoso JC, Cork LC, Coyle JT, Price DL Synaptic neurochemical alterations associated with neuronal degeneration in an inherited cerebellar ataxia of Gordon Setters. J Neuropathol Exp Neurol 1984; 43: 580-591.

50. Twiss JL, Baisch M, Horoupian DS. Neonatal encephalopathy with neuronal vacuolar degeneration. Acta Neuropathol 1993; 86: 536-541.

51. Uusisaari MY, Knopfel T. Diversity of neuronal elements and circuitry in the cerebellar nuclei. Cerebellum 2012; 11: 420-421.

52. van den Ingh TS, Mandigers PJ, van Nes JJ. A neuronal vacuolar disorder in young rottweiler dogs. Veterinary Record 1998; 142: 245-247.

53. Zerial M, McBride H. Rab proteins as membrane organizers. Nat Rev Mol Cell Biol 2001; 2: 107-117. 